\title{
State-dependent regulation of cortical activity by cortisol: An EEG study
}

\author{
Mattie Tops ${ }^{\mathrm{a}, \mathrm{b}, *}$, Jacobien M. van Peer ${ }^{\mathrm{a}, \mathrm{b}, \mathrm{c}}$, Anne E. Wester ${ }^{\mathrm{a}, \mathrm{b}, \mathrm{d}}$, \\ Albertus A. Wijers ${ }^{\text {a }}$, Jakob Korf ${ }^{b}$ \\ ${ }^{a}$ Department of Experimental and Work Psychology, University of Groningen, Grote Kruisstraat 2/1, 9712 TS Groningen, The Netherlands \\ ${ }^{\mathrm{b}}$ Department of Psychiatry, University Medical Center Groningen, Hanzeplein 1, 9700 RB Groningen, The Netherlands \\ ${ }^{\mathrm{c}}$ Section of Clinical and Health Psychology, University of Leiden, P.O. Box 9555, 2300 RB Leiden, The Netherlands \\ ${ }^{\mathrm{d}}$ Department of Psychopharmacology, Utrecht Institute of Pharmaceutical Sciences, University of Utrecht, \\ P.O. Box 80082, 3508 TB Utrecht, The Netherlands
}

Received 6 March 2006; received in revised form 4 May 2006; accepted 17 May 2006

\begin{abstract}
In the present study we investigated the effects of cortisol administration on EEG activity in eight healthy volunteers. We administered 35 mg of cortisol in a within-subjects double-blind placebo-controlled design. Cortisol administration caused a global decrease in cortical activity except for an increase frontally at the left, resulting in a significant change in frontal asymmetry. This pattern of results is almost the exact mirror image of one of our previous studies. Comparing subjective activation measures from the present and previous study showed that activation was substantially higher in the present study that was performed in formal testing conditions involving venipuncture, compared to the previous EEG study that was performed in non-formal testing conditions. However, the direction of the present cortisolinduced change in frontal activity asymmetry is consistent with the cortisolinduced change in asymmetric rotation behavior that we recently reported in similar testing conditions. We also found indications that, in contrast to the effects on the EEG measures, effects of cortisol administration on subjective anxiety and plasma oxytocin levels may be sex-dependent. These results are preliminary because of the post-hoc nature and the small number of subjects in the present study. However, they are in line with recent findings by others, suggesting that the effects of cortisol on cortical activity and subjective activation are state dependent and are influenced by testing conditions.
\end{abstract}

(C) 2006 Elsevier Ireland Ltd. All rights reserved.

Keywords: Cortisol; EEG; Frontal asymmetry; Venipuncture stress; Testing conditions; Alpha power

The hormone cortisol has a wide range of physiological functions, including the mobilization of resources and stressprotective down-regulation of other physiological systems [3]. Effects of cortisol are context- and state-dependent [3]. Reuter studied the effects of cortisol administration on self-reported mood and activation under stress (violent film) and nonstress (neutral film) conditions [13]. Reuter summarized his results as indicating an adaptive activating effect of cortisol in conditions in which there is a loss of activity due to lack of stimulation since it prevents a further decrease in activity, whereas in stress conditions the organism is protected from overactivation. Indeed, recently it was found that acute cortisol treatment attenuated increases in fear in response to phobia-related stimuli in both a group of subjects with social phobia and a group with spi-

\footnotetext{
* Correspondence to: NeuroInformatics Center, University of Oregon, OR 97403, USA. Tel.: +1 541 6862903; fax: +1 5416877963.

E-mail address: mtops@darkwing.uoregon.edu (M. Tops).
}

der phobia [15]. Similarly, mere testing conditions modulate the effects of cortisol on memory retrieval. In line with observations in rats, Kuhlman and Wolf observed that cortisol administration impaired memory retrieval in a formal testing condition, while it had no impact on retrieval in a very relaxed non-formal testing condition [9].

These results support the idea that a certain emotional arousal is a prerequisite for certain cortisol effects to occur. Indeed, Elzinga and Roelofs found that effects of cortisol administration on working memory depended on sympathetic activation [5]. Abercrombie et al. found that larger stress-induced cortisol elevations were related to memory facilitation only in subjects who reported stress-related negative affect [1]. Alternatively, differential activation by cortisol of mineralocorticoid receptors (MR) and glucocorticoid receptors (GR) may be involved. For instance, in hippocampal CA1 neurons, activation of MR maintained neuronal excitability and activation of GR suppressed excitability transiently raised by excitatory stimulation [8]. In a very relaxed condition cortisol will tend to mainly activate MR 
receptors, while with increasing stress more GR receptors will be activated [8].

In a recent study that was performed in a relaxed, non-formal testing condition, we investigated the acute effects of cortisol (35 mg) administration on cortical activity as measured by EEG alpha power suppression in male volunteers [18]. Cortisol compared to placebo globally increased activity, including at right frontal sites but except for at the left lateral frontal site. However, in another recent study, which was performed in a formal hospital environment and included venipuncture, we found that $35 \mathrm{mg}$ of cortisol modulated a behavioral measure (asymmetrical turning behavior) indicative of reduced right frontal activity [17]. Also in the present study we measured EEG activity after administration of $35 \mathrm{mg}$ cortisol or placebo in healthy volunteers. In comparison to our previous EEG study, this time both male and female volunteers were included, and capsule administration took place at 10:25. In the previous EEG study the capsule was administered at 9:00 to one half of the volunteers, and at 13:00 to the other half of the volunteers; these different times of administration did not seem to make a difference in the results. Another difference between the present and previous EEG study is that in the present study blood sampling by venipuncture took place. Venipuncture and catheterization are potent psychological and physiological stressors, and have been found to increase cortisol levels for between 1 and $2 \mathrm{~h}$ [14]. Moreover, whereas in the previous EEG study the subjects were measured in between a lengthy performance of a monotonous cognitive task in a dimly lit room in the relaxed, non-formal environment of the psychology department, in the present study the subjects were measured in the formal environment of a university hospital in a fully lit room, similar to our behavioral study. In the previous EEG study the experimenter observed the subject by camera from outside the room, while in the present study and the behavioral study, one or two experimenters were present in the room during measurements.

In the present study, the effects of cortisol administration on cortical activity were almost exactly opposite to the previous EEG study. Because the most notable difference between this and the behavioral study compared to the first EEG study seems to be the activating level of the experimental conditions, apart from presenting the results of the present study, we will also compare the subjective reports of activation with those from the previous EEG study. Although this comparison is post-hoc, it may inform future studies in pointing to dependency on testing conditions of the effects of cortisol administration on cortical activity. As the present study, similar to the previous studies, intended to investigate the effects of cortisol administration on frontal activity asymmetry, we will also include hemisphere as a factor in our analyses. We also measured plasma levels of cortisol, adrenocorticotropic hormone (ACTH), vasopressin and oxytocin. Plasma levels of vasopressin and oxytocin have been related to cortical activity and subjective activation [11].

Five females aged 37-51 (M=41) and five males aged 38-55 $(\mathrm{M}=47)$ participated who were right-handedness and had no personal history of psychiatric, metabolic, or neurological disorders. The female subjects did not take oral contraceptives, were not pregnant or breast feeding. The study was approved by the Medical Ethical Committee of the University Hospital of Groningen and all subjects provided written informed consent. Selection procedure, level of education and inclusion criteria were similar to the previous EEG study, in which the mean age of the participants was 28 years.

We used a within-subject double-blind design. Placebo and cortisol sessions, the order of which was counterbalanced, were separated by approximately 1 week. Participants came to the experimental room at 10:15. At 10:25 (T0) participants ingested a non-labelled capsule, containing either $35 \mathrm{mg}$ hydrocortisone or placebo. At 11:30 blood samples were taken. At 12:45 (T1) participants started to work on a free recall task that took about $25 \mathrm{~min}$. Directly before (starting $1 \mathrm{~h}$ after venipuncture) and directly after this task resting EEG was recorded. The Profile of Mood States (POMS) short version [20] was filled out by the subjects at T0, T1 and after the last EEG measurement (T2). The POMS measures vigor, fatigue, depression, tension and anger. Additionally, the state version of the State-Trait Anxiety Inventory (STAI) was filled out at T0 and T2.

Plasma concentrations of cortisol and ACTH were determined using a radio immunoassay. Vasopressin was extracted from the plasma using Sep-pak C 18 cartridges. The extracts were analyzed with a radioimmunoassay (MP-products, Amersfoort). All analyses were performed at the University Hospital of Groningen. Oxytocin levels were measured by radioimmunoassay at the Swedish University of Agricultural Sciences in Uppsala at the Department of Animal Physiology. The antibody $\mathrm{KA}_{19}$ was used for the analysis (Milab, Sweden). The limit of detection was $4.68 \mathrm{pmol} / 1$ and interassay coefficients of variation low $22.06 \% \mathrm{CV}=9.57$; medium $37.83 \% \mathrm{CV}=8.81$; high $529.8 \% \mathrm{CV}=6.81$; intrassay $\% \mathrm{CV}<10$ conc $=20.90-1026$ [16].

EEG signals were recorded using the Onyx recording program (Version 1.02: Silicon Biomedical Instruments BV 2000), using an average reference. Horizontal EOG was measured with Sn electrodes on the outer canthi. Vertical EOG was measured from the upper and lower edge of the left orbit. A ground electrode was connected to the participants' sternum. Electrode impedance was held under $5 \mathrm{k} \Omega$. EEG and EOG were sampled at $256 \mathrm{~Hz}$.

Each measurement consisted of a series of eight 1-min recording periods, during which EEG was recorded while the participant sat quietly with eyes opened and closed alternately in counterbalanced orders. With eyes opened, participants were instructed to look at a fixation point in front of them.

EEG signals were processed off-line, with the Vision Analyzer program (Version 1.03: Brain Products GmbH 2000). Signals were re-referenced to an average-ears reference, and the data of each 1-min recording period were segmented into 2-s segments, with $50 \%$ overlap. Automatic ocular correction and automatic artifact rejection were performed on these segments. Subsequently, we performed a Fast Fourier Transform (half spectrum method) on these data with a resolution of $0.5 \mathrm{~Hz}$ and a Hamming window of $100 \%$ length, resulting in power estimates for each $0.5 \mathrm{~Hz}$ bin. Next, data was averaged across each block. We visually inspected the data to exclude blocks that contained visible artifacts. By also removing the same block in the other session, we saw to it that for each participant an equal number of 
'eyes-closed' and 'eyes-open' blocks remained in both sessions. At least four blocks per session remained. We ln transformed the average power values of the frequencies $8-13 \mathrm{~Hz}$, resulting in $\ln$ alpha power density $\left(\mu \mathrm{V}^{2} / \mathrm{Hz}\right)$. Statistical analyses were performed on the average power density across all recording periods, per participant per session.

Due to technical problems, we failed to obtain usable EEG data from one male and one female subject. Similar to our previous EEG study, as the effects of cortisol treatment did not interact with 'eyes open' versus 'eyes closed' conditions, we collapsed the data across these conditions. We conducted a MANOVA repeated measures analysis with Capsule (cortisol, placebo), Hemisphere (left, right), Caudality (frontal, posterior), Laterality (middle, lateral) and electrode as within-subject factors. The grouping of electrodes was as follows: left mid-frontal (Fp1, F3); left lateral frontal (F7, T7); left mid-posterior (C3, P3); left lateral posterior $(\mathrm{P} 7, \mathrm{O} 1)$; and similarly on the right side. Decreases in alpha power are indicative of increased activity [10].

A capsule by hemisphere interaction $(F(1,7)=7.66, p=.028)$ indicated a global decrease in activity that was more pronounced over the right hemisphere than over the left hemisphere (see Fig. 1). There was an interaction of capsule $\times$ hemisphere $x$ caudality $\times$ laterality $(F(1,7)=6.67, p=.036)$ originating from a capsule by hemisphere interaction on the frontal electrodes

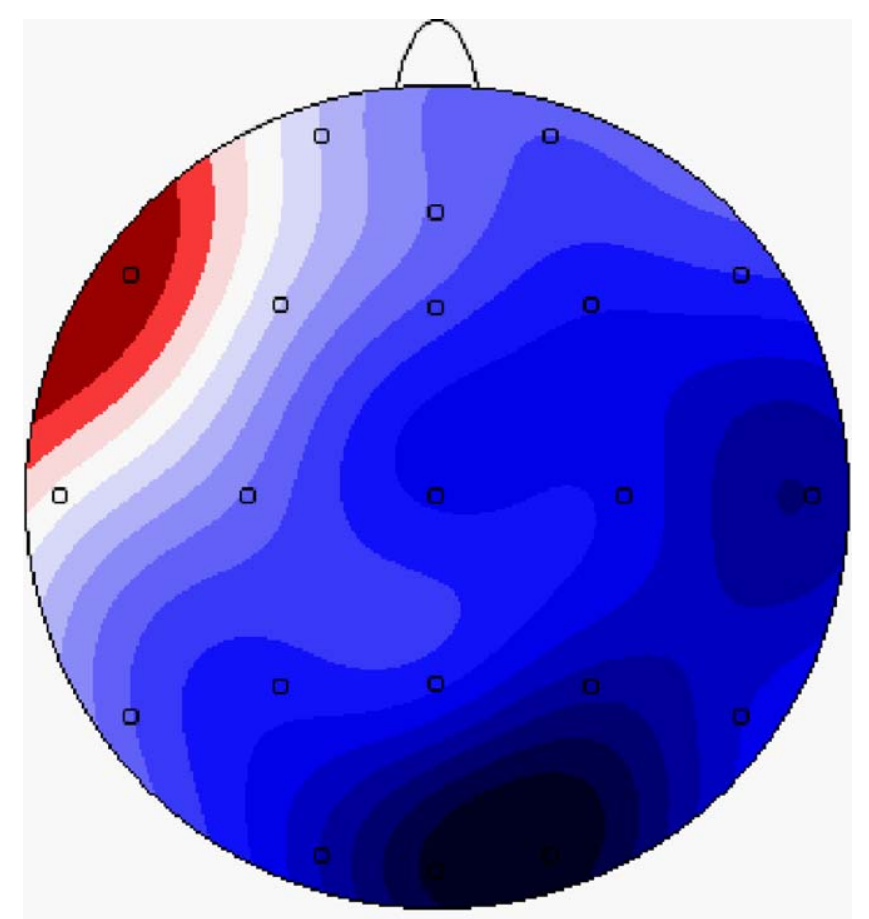

$8.0-13.0 \mathrm{~Hz}$

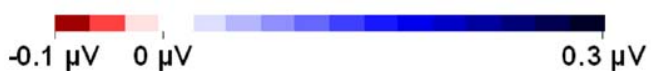

Fig. 1. Interpolation by spherical splines of ln-transformed alpha $(8-13 \mathrm{~Hz})$ power in $\mu \mathrm{V}^{2}$; see [18]. Power in the placebo condition is subtracted from power in the cortisol condition. This means that blue colors reflect more alpha power, indicative of less activity [10], in the cortisol condition compared to placebo. which was significant lateral frontally $(F(1,7)=18.62, p=.004)$. The participants tended to display less frontal activity left $\left(\mathrm{M}=-.82 \mu \mathrm{V}^{2} / \mathrm{Hz}\right.$, S.D. =1.21) compared to right in the placebo condition $(\mathrm{M}=-.90$, S.D. $=1.16)(F(1,7)=4.31$, $p=.076)$; in the cortisol condition this activity increased on the left $(\mathrm{M}=-.96$, S.D. $=1.18)$ and decreased on the right $(\mathrm{M}=-.79$, S.D. $=1.25)$ (see Fig. 1).

Except at the left lateral frontal electrodes, activity decreased at all electrodes. At local electrodes the decreased activity after cortisol administration was significant for electrodes $\mathrm{P} 4$ and $\mathrm{O} 2$ $(p s<.05)$ and a trend for electrodes F4, T8, C4 and O1 $(p s<.10)$. When looking at the male and female subjects separately, both sexes showed a similar cortisol-induced decrease of cortical activity and an increase at the left lateral frontal sites.

We ln-transformed cortisol and ACTH values to better approach a normal distribution. A significant capsule effect on plasma cortisol levels (placebo: $\mathrm{M}=248 \mathrm{nmol} / \mathrm{l}$, S.D. $=89$; cortisol: $\mathrm{M}=1057$, S.D. $=313 ; F(1,8)=114.33, p<.001)$ indicated a large increase in levels of cortisol after administration of cortisol. Plasma levels of ACTH were found to be significantly lower after cortisol administration than in the placebo condition (placebo: $\mathrm{M}=24.0 \mathrm{ng} / \mathrm{l}$, S.D. $=12.3$; cortisol: $\mathrm{M}=15.5$, S.D. $=8.7 ; F(1,8)=14.04, p=.008)$, demonstrating negative feedback. Administration of cortisol did not suppress vasopressin (placebo: $\mathrm{M}=1.11 \mathrm{ng} / \mathrm{l}, \mathrm{S} . \mathrm{D} .=49$; cortisol: $\mathrm{M}=1.19$, S.D. $=.54)$.

The subjects of the present study were part of a larger, non-EEG study on the effects of cortisol administration on plasma oxytocin levels that will be presented elsewhere. Here, we present the oxytocin data of the subjects that participated in the EEG measurements as they may be relevant for the EEG findings. The plasma oxytocin levels demonstrated a significant sex by capsule interaction $(F(1,8)=5.37, p=.049)$. In the placebo condition oxytocin levels tended to be lower in females $(\mathrm{M}=42.7 \mathrm{pmol} / \mathrm{l}, \mathrm{S} . \mathrm{D} .=12.3)$ compared to males $(\mathrm{M}=64.6$, S.D. $=7.3 ; F(1,8)=3.54, p=.097)$. In females levels increased with cortisol administration $(\mathrm{M}=49.3$, S.D. $=11.8$; $F(1,4)=8.22$. $p=.050)$. In males there was a nonsignificant decrease $(\mathrm{M}=53.2$, S.D. $=11.6 ; p>.10)$.

MANCOVA repeated measures with the STAI-state scores at baseline as covariate showed an interaction between sex and capsule $(F(1,7)=7.31, p=.030)$. In the placebo condition females tended to report more anxiety (females: $\mathrm{M}=29.8$, S.D. $=2.8$; males: $\mathrm{M}=26.4$, S.D. $=2.8 ; F(1,26)=3.02, p=.10)$. In females anxiety decreased with cortisol administration (cortisol: $\mathrm{M}=26.1$, S.D. $=2.8 ; F(1,3)=11.88, p=.041)$, whereas in males cortisol increased anxiety $(\mathrm{M}=28.0$, S.D. $=2.8$; $F(1,3)=37.31, p=.009)$.

Most results regarding the POMS mood scales were not significant. However, the size of the present study is small, and we present the effects of cortisol on mood scores of the female subjects elsewhere as part of a larger non-EEG study. The pattern of results in the present study is the same as in the larger study: the female subjects showed a pronounced increase in fatigue and decrease in vigor from before the start to after finishing the testing session in the placebo condition but not in the cortisol condition. The male subjects show a different pattern as 
described below. There were no differences between males and females in baseline (T0) levels of any of the mood scales.

Both in the present and previous EEG study, mood was measured by the POMS (but not the STAI in the previous study) at T0, T1 and T2. This allows us to compare the activational properties of the testing conditions in both studies. Vigor scores were higher in the present study $(M=14.2$, S.D. $=2.9)$ compared to the previous study (morning: $\mathrm{M}=9.1, \mathrm{~S} . \mathrm{D} .=3.0$; afternoon: $\mathrm{M}=9.3$, S.D. $=3.0 ; F(1,19)=10.78, p=.004)$. Fatigue scores were lower in the present study $(\mathrm{M}=.5, \mathrm{~S} . \mathrm{D} .=1.8)$ compared to the previous study (morning: $\mathrm{M}=3.5, \mathrm{~S} . \mathrm{D} .=1.7$; afternoon: $\mathrm{M}=3.6$, S.D. $=1.7 ; F(1,19)=9.00, p=.007)$. As only male subjects were tested in the previous study, and in the present study, after cortisol administration, vigor scores increased in females, but decreased in males, we compared the male subjects of both studies. Compared to the decrease in the present study, cortisol increased vigor in the previous study (MANCOVA: study $\times$ capsule $F(1,13)=4.68, p=.052$, see Fig. 2 ). Regarding the other mood scales no effects approached significance.

In the present study cortisol administration caused a global decrease in cortical activity except for an increase frontally at the left, resulting in a significant change in frontal asymmetry. This pattern of results is almost the exact mirror image of the results from our previous study [18]. Similarly, while in our previous study we found that in the placebo condition activity was significantly higher at the lateral frontal electrodes on the left compared to on the right side, in the present study there was a trend in the placebo condition of an opposite asymmetry at the lateral frontal electrodes. Notice that the present results are similar to the previous EEG results, in that the largest changes in asymmetry were found at the lateral frontal sites, and the change at the right lateral frontal site was in the same direction as a global change in activity, while only at the left lateral frontal site there was a nonsignificant change in the other direction. The direction of cortisol-induced change in frontal activity asymmetry is consistent with the cortisol-induced change in asymmetric rotation behavior, that was found in similar testing conditions as in the present study [17].

Consistent with the possibility that the opposite effects of cortisol administration on cortical activity are mediated by dif-

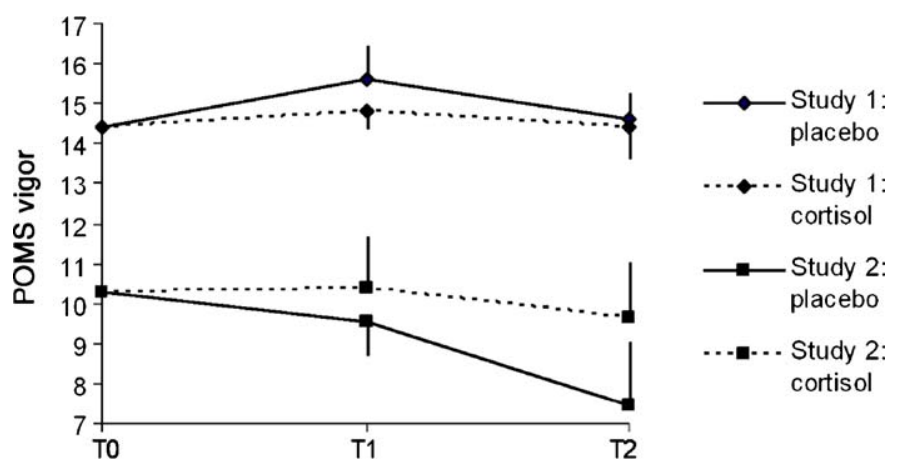

Fig. 2. Mean POMS vigor scores and S.E.s for the treatment and placebo conditions of the present formal, high activation study 1 (only the males) and the previous, low activation study 2. Means at T1 and T2 are adjusted for differences at T0; means at T0 are averaged over both sessions. ferent arousing qualities of the testing conditions, the subjects of the two EEG studies reported significantly different levels of subjective activation. In the formal testing conditions, including venipuncture, of the present study, self-reported activation was higher compared to in the non-formal testing conditions of the previous EEG study. For the male subjects, intake of cortisol decreased activation in the present, high activation study, compared to an increase in activation in the previous, low activation study. Although this effect was borderline significant, it replicates the similar findings by Reuter who tested only male subjects [13].

We found sex differences in the effects of cortisol administration on plasma oxytocin levels and state anxiety scores. In males oxytocin levels decreased and anxiety increased, in females oxytocin levels increased and anxiety decreased. Although the analyses of sex differences are based on very small groups, the opposite changes in oxytocin levels and state anxiety are consistent with the anxiolythic effects of oxytocin [19]. Moreover, increasing plasma oxytocin levels by bolus injection decreases subjective deactivation, fatigue, benumbing, but also arousal and anger, in male subjects [11]. This is consistent with the hypothesis that oxytocin promotes the state of "calm and connection" to facilitate social interaction [19]. It is also the same pattern of decreased subjective deactivation and decreased subjective overactivation as reported by Reuter after administration of cortisol to male subjects [13]. This suggests that changes in plasma oxytocin levels, inductions of which have also been inconsistently related to cortical activity [11], may be mediating some of the effects of cortisol administration, perhaps including the opposite changes between males and females in anxiety, and state-dependent effects. We are not aware of an explanation of the sex difference in treatment response in oxytocin levels and anxiety, but not cortical activity. Perhaps a larger replication study can try to address this issue.

Another candidate mechanism for mediating the statedependent effects of cortisol involves the locus ceruleus/ norepinephrine (NE) system. Cortisol serves as a negative feedback control on this system [2]. The NE arousal system is relatively right lateralized [4]. Serotonin effects on the NE system seem to depend on the baseline activity level in the NE system: $\mathrm{NE}$ activity increases when it is low at baseline, but it decreases when it is high at baseline [7]. This means that an indirect effect of cortisol administration on activity in the NE arousal system through an effect on serotonergic activity [12] may hypothetically explain the presently reported state-dependent effects on cortical activity. As the locus ceruleus/NE system participates in the initiation of fight/flight and cortisol responses, counter regulation of increased activity in this system by cortisol may be part of a functional regulatory loop [2]. Other mechanisms that may explain the present results were mentioned in the introduction.

The inclusion of female participants and older mean age in the present study, or the different time of day of ingestion of the capsule, are not likely explanations of the opposite results of the present and previous EEG study. The effects of cortisol administration on alpha power were similar for our female and male participants. No effects are known to us of variations in age between 20 and 55 years that would offer a plausible expla- 
nation for the present results. Moreover, age did not correlate with outcome measures in either study. In our previous study the effects of cortisol administration were similar in participants who ingested the capsule at 9:00 compared to participants who ingested the capsule at 13:00; in the present study the capsule was ingested at a time halfway in between those two times.

An alternative explanation for the presented results is that effects of cortisol administration do not really depend on testing conditions, but that cortisol administration prevents testing condition from having effects on the outcome measures. It may be hypothesized that the sustained, very high cortisol levels after cortisol administration may exclude both MR and GR receptors from further activation, thereby decreasing the responsivity of several brain systems. Both decreases of cortical activity (and of subjective activation) by non-formal testing conditions and lack of stimulation, and increases in cortical activity (and subjective activation) by formal testing conditions would not take place after cortisol administration, to the extent that cortisol, or other systems that are rendered unresponsive by high cortisol levels, are normally involved in the modulation of such activity.

The effects on frontal activity asymmetry also seemed to depend on testing conditions. Left and right frontal activity asymmetry have been related to approach and avoidance motivation, respectively, and to psychopathology [6]. The present finding of cortisol-induced reduced right frontal activity is consistent with results we recently reported [17]. In that study, which was performed in the formal hospital environment and included venipuncture, we found that $35 \mathrm{mg}$ of cortisol modulated a behavioral measure (asymmetrical turning behavior) indicative of reduced right frontal dopaminergic activity. However, it remains an open question if cortisol regulates dopaminergically modulated frontal activity asymmetry that is related to approach motivation [18]. Future studies on the effects of cortisol on frontal activity asymmetry should include behavioral measures of approach and avoidance motivation to answer this question.

The present results suggest that the effects of cortisol on cortical activity, similar to the effects on activation [13] and memory retrieval [9], are state dependent and are influenced by testing conditions. This conclusion is preliminary because of its posthoc nature and the limited number of subjects. However, the presented results caution similar studies in the future to carefully take into account testing conditions. Moreover, state-dependent effects of cortisol on cortical activity and subjective activation deserve further study in the light of a recent report that cortisol administration protects against the fear arousing effect of phobia-related stimuli in subjects with social phobia or spider phobia [15].

\section{References}

[1] H.C. Abercrombie, N.S. Speck, R.M. Monticelli, Endogenous cortisol elevations are related to memory facilitation only in individuals who are emotionally aroused, Psychoneuroendocrinology 31 (2006) 187-196.

[2] A. Calogero, W.T. Gallucci, P.W. Gold, G.P. Chrousos, Multiple feedback regulatory loops upon rat hypothalamic corticotropin releasing hormone secretion, J. Clin. Invest. 82 (1988) 767-774.

[3] E.R. De Kloet, M.S. Oitzl, M. Joëls, Stress and cognition: are corticosteroids good or bad guys? Trends Neurosci. 22 (1999) 422-426.

[4] J. Dien, Different lateralization of trait anxiety and trait fearfulness: evoked potential correlates, Pers. Individ. Dif. 26 (1999) 333-356.

[5] B.M. Elzinga, K. Roelofs, Cortisol-induced impairments of working memory require acute sympathetic activation, Behav. Neurosci. 119 (2005) 98-103.

[6] E. Harmon-Jones, Clarifying the emotive functions of asymmetrical frontal cortical activity, Psychophysiology 40 (2004) 838-848.

[7] R.J. Hedaya, Understanding Biological Psychiatry, W.W. Norton, New York, 1996, p. 63.

[8] M. Joels, E.R. De Kloet, Effects of glucocorticoids and norepinephrine on the excitability in the hippocampus, Science 245 (1989) 1502-1505.

[9] S. Kuhlmann, O.T. Wolf, A non-arousing test situation abolishes the impairing effects of cortisol on delayed memory retrieval in healthy women, Neurosci. Lett. 399 (2006) 268-272.

[10] H. Laufs, A. Kleinschmidt, A. Beyerle, E. Eger, A. Salek-Haddadi, C. Preibisch, K. Krakow, EEG-correlated fMRI of human alpha activity, Neuroimage 19 (2003) 1463-1476.

[11] R. Pietrowsky, D. Braun, H.L. Fehm, P. Pauschinger, J. Born, Vasopressin and oxytocin do not influence early sensory processing but affect mood and activation in man, Peptides 12 (1991) 1385-1391.

[12] R.J. Porter, P. Gallagher, S. Watson, A.H. Young, Corticosteroidserotonin interactions in depression: a review of the human evidence, Psychopharmacology 173 (2004) 1-17.

[13] M. Reuter, Impact of cortisol on emotions under stress and nonstress conditions: a pharmacological approach, Neuropsychobiology 46 (2002) $41-48$.

[14] R.M. Rose, M.W. Hust, Plasma cortisol and growth hormone responses to intravenous catheterization, J. Hum. Stress 1 (1975) 22-36.

[15] L.M. Soravia, M. Heinrichs, A. Aerni, C. Maroni, G. Schelling, U. Ehlert, B. Roozendaal, D.J.-F. de Quervain, Glucocorticoids reduce phobic fear in humans, Proc. Natl. Acad. Sci. U.S.A. 103 (2006) 5585-5590.

[16] S. Stock, K. Uvnas-Moberg, Increased plasma levels of oxytocin in response to afferent electrical stimulation in sciatic and vagal nerves and in response to touch and pinch in anaesthetized rats, Acta Physiol. Scan. 132 (1988) 29-34.

[17] M. Tops, A.A. Wijers, T. Koch, J. Korf, Modulation of rotational behavior in healthy volunteers by cortisol administration, Biol. Psychol. 71 (2006) 240-243.

[18] M. Tops, A.A. Wijers, A. van Staveren, K.J. Bruin, J.A. den Boer, T.F. Meijman, J. Korf, Acute cortisol administration modulates EEG alpha asymmetry in volunteers: relevance to depression, Biol. Psychol. 69 (2005) 181-193.

[19] K. Uvnäs-Moberg, I. Arn, D. Magnusson, The psychobiology of emotion: the role of the oxytocinergic system, Int. J. Behav. Med. 12 (2005) $59-65$.

[20] F.D.M. Wald, G.J. Mellenberg, De verkorte versie van de Nederlandse vertaling van de Profile of Mood States (POMS), Ned. Tijdschr. Psychol. 45 (1990) 86-99. 